\title{
Accumulation Ratio Cmin Normalized by Dose
}

National Cancer Institute

\section{Source}

National Cancer Institute. Accumulation Ratio Cmin Normalized by Dose. NCI Thesaurus.

Code C132438.

The minimum concentration at steady state divided by the minimum concentration during the initial dosing interval, each divided by the associated dose. (CDISC) 